\title{
Kidney Mixed Epithelial and Stromal Tumor Family
}

National Cancer Institute

\section{Source}

National Cancer Institute. Kidney Mixed Epithelial and Stromal Tumor Family. NCI

Thesaurus. Code C158046.

A family of tumors ranging from predominantly cystic tumors (adult cystic nephromas)

to tumors that are variably solid (mixed epithelial and stromal tumors) and contain biphasic epithelial and stromal components with spindle stroma, glands, and cysts. Most of these tumors are benign. (WHO 2016). 